\title{
Big data and innovative bioinformatics approaches in personalized genomic medicine
}

\author{
Joris A Veltman ${ }^{1}$ \\ ${ }^{1}$ Biosciences Institute, Faculty of Medical Sciences, Newcastle University
}

\begin{abstract}
The field of human genetics has been radically changed by the introduction of massive parallel sequencing, also called next generation sequencing, approaches. Instead of studying a single gene or a few genetic variants, nowadays we can study genetic variation present in all genes and even throughout the entire human genome. For the first time in history, we can really study what makes us unique and use that to explain differences in for example disease susceptibility or response to treatment. In rare disease, genetics research is essential to identify the molecular diagnosis that provides the basis for a personalized patient management approach. It allows for more precise answers about the underlying cause and family recurrence risk, but also aids in optimizing treatment plans aimed at reducing co-morbidities and providing information about potential drugs or participation in drug trials, with an increasing number focused on gene therapy. These high-throughput sequencing technologies generate enormous amounts of data in order to assemble a genome and identify all of the variation present at different levels, from single nucleotide variations to chromosomal abnormalities. In addition, a genome sequence of a person in itself is not very useful. Value is derived from annotation of all the variation, and integration of the genome sequence with information about the patient involved (clinical information, disease-specific information, family history) as well as biological information (gene as well as variant-specific information, including population variation frequency, pathogenicity predictions, gene-expression information, etc). In this presentation, I will give an overview of the impact of genomics on the diagnosis of patients with rare developmental disorders and fertility disorders. I will highlight the importance of innovative bioinformatics approaches to detect and interpret genetic variation in a clinical context. Also, I will highlight some of the challenges that individual research and diagnostics units face in dealing with the data generated, discuss some of the ethical/privacy issues related to these approaches and discuss some of the latest genomics technologies being developed and validated.
\end{abstract}

